\title{
PROPOSTA DE MODELO DE AVALIAÇÃO DA QUALIDADE DIRECIONADO AO SISTEMA ÚNICO DE SAÚDE
}

\section{Alice Silau Amoury Neta}

aliceamoury@outlook.com.br Universidade do Estado do Pará UEPA, Marabá, Pará, Brasil.

Brenna Cecília Sousa Ferreira brennacecilia.ferreira@gmail.com Universidade do Estado do Pará UEPA, Marabá, Pará, Brasil.

\section{Rodrigo Rangel Ribeiro} Bezerra

rodrigo.bezerra@uepa.br Universidade do Estado do Pará UEPA, Marabá, Pará, Brasil.

Leonardo Breno Pessoa Silva leonardobrenopessoa@hotmail. com

Universidade Tecnológica Federal do Paraná - UTFPR, Ponta Grossa, Paraná, Brasil.

\section{RESUMO}

A avaliação da qualidade direciona esforços para áreas críticas, principalmente quando se fala em gestão de saúde pública, que necessita ser eficiente para o atendimento da população. Neste sentido, ferramentas direcionadas à avaliação da qualidade nesse setor mostram-se pertinentes. Este trabalho teve como objetivo propor um modelo de avaliação da qualidade direcionado ao Sistema Único de Saúde, desenvolvido com base no instrumento Service Quality, e considerando aspectos concernentes ao Institute of Medicine, Programa Nacional de Avaliação dos Serviços de Saúde e à legislação correspondente. Dois instrumentos de coleta foram desenvolvidos e validados estatisticamente por meio do coeficiente alfa para composição do modelo referido. Ao considerar a importância relativa das dimensões sob perspectiva dos três públicos (usuários, profissionais e gestores), a aplicação do modelo SUSSQUAL se mostrou útil para orientação de gestores e/ ou profissionais interessados nos processos de tomada de decisões, e no delineamento de estratégias que possuam como fins a elevação qualitativa das atribuições do setor de saúde pública.

Palavras-chave: Saúde Pública; Sistema Único de Saúde; Avaliação da Qualidade; Service Quality; SUSSQUAL. 


\section{INTRODUÇÃO}

Com o atual panorama de crise econômica em que o Brasil se encontra, vale ressaltar o aumento da relevância dos trabalhos desenvolvidos na busca pela otimização dos recursos. Em todas as organizações há a necessidade de indicadores tanto para avaliação externa quanto para o processo de gestão. Além disso, é preciso traçar indicadores de desempenho que respondam aos objetivos estratégicos, imprimindo a implementação da estratégia organizacional (Vecina Neto; Malik, 2012).

As organizações hospitalares, em específico, desempenham determinada pluralidade processual que as configura como organizações complexas e necessitam ser encaradas como tais. Como enfatizado por Vecina Neto e Malik (2012), essa complexidade advém da coexistência de inúmeros processos assistenciais e administrativos, linhas de produções simultâneas e descentralização do processo de decisão. Assim, é de conhecimento que o estudo acerca dos aspectos qualitativos referentes à prestação de serviço no setor público de saúde é relevante, haja vista que se trata de um setor complexo. Mezomo (2001) afirma que essa complexidade é ocasionada por uma série de fatores internos e ambientais, que acabam comprometendo a qualidade do serviço.

Vale memorar-se que a legislação do Sistema Único de Saúde (SUS) normatiza a saúde como um direito de todos os cidadãos e dever do Estado. Porém, Mendes (2002) enfatiza que o sistema que deveria ser único tornou-se plural, e hoje é composto por três grupos: o sistema público, o sistema de atenção médica supletiva, (referente aos planos privados) e o sistema de desembolso direto. Infere-se que essa segmentação do sistema tenha ocorrido em decorrência da ineficiência de recursos públicos para atendimento satisfatório à elevada demanda agregada ao sistema, que, por sua vez, necessitou migrar-se gradativamente às outras esferas de prestação de serviços de saúde para que sua necessidade viesse a ser suprida.

Pesquisa realizada pelo Instituto Trata Brasil e Instituto Brasileiro de Opinião Pública e Estatística (2012) estipulou que cerca de $61 \%$ dos brasileiros consideram a saúde com a área mais problemática do País. Trata-se de um referendo sobre o sistema público de saúde nacional, já que 3 de cada 4 brasileiros dependem dele (Prates, 2012). De acordo com o Conselho Federal de Farmácia (2015), o Brasil é um dos países que apresentam menor investimento em saúde pública. Os dados mostram que o governo brasileiro investe $4,7 \%$ do Produto Interno Brasileiro (PIB) em saúde, índice muito inferior aos gastos de Canadá, França, Suíça e Reino Unido, onde os porcentuais de investimento variam de 7,6\% a 9,0\%.

Além do subfinanciamento, identifica-se um quantitativo crescente de usuários criteriosos com a qualidade dos ser- viços de saúde. Neste sentido, ferramentas direcionadas à avaliação da qualidade nesse setor mostram-se pertinentes. É válido ressaltar que o tema qualidade de serviços tem apresentado forte crescimento nas últimas décadas, dada a sua importância para a economia. São vários os modelos de avaliação da qualidade passíveis de utilização no ambiente de serviços, mas que demandam customização (Batalha, 2008).

O Service Quality (SERVQUAL), desenvolvido por Parasuraman et al. (1988), é conceituado por Ferreira (2015) como um instrumento designado para medir a qualidade do serviço prestado a partir das percepções dos clientes. De acordo com Sousa et al. (2011), o SERVQUAL surgiu para tentar resolver as seguintes situações: a revisão dos estudos que investigaram a qualidade de serviços na compra de bens e serviços; rever os relatórios dos insights obtidos em investigação exploratória extensiva da qualidade em quatro empresas de serviços; desenvolver um modelo de qualidade em serviços; e propor condições para desenvolvimento de futuros estudos sobre a qualidade.

A relevância desse estudo justifica-se por sua contribuição no campo científico, uma vez que sugere um modelo de mensuração de qualidade customizado para aplicação no setor de serviços ofertados pelo SUS, a fim de que propicie avanços na área científica e atue como método para desenvolvimento de pesquisas científicas futuras eficazes no setor ao qual se destina, que é pouco explorado pela literatura.

O objetivo deste estudo é propor um modelo de avaliação da qualidade direcionado aos serviços ofertados pelo SUS, com base no modelo SERVQUAL. Para isso, é necessário listar as utilizações do modelo SERVQUAL no setor de saúde e as variações dessa ferramenta, além de desenvolver um modelo adaptado com aspectos do Institute of Medicine (IOM), Programa Nacional de Avaliação dos Serviços de Saúde (PNASS), legislações pertinentes e validar o modelo matematicamente. O IOM foi escolhido por se tratar de uma organização independente que trata de assuntos relevantes à saúde e à medicina, estimulando ações positivas ao setor. A organização conduz estudos e emite relatórios que fornecem informações imparciais e qualificadas para os responsáveis por tomadas de decisões políticas e para a população (Allen Jr., 2016). O PNASS constitui-se uma ferramenta para avaliar estabelecimentos de saúde e atenção especializada, buscando levantar indicadores pertinentes a serem trabaIhados para propostas de melhoria (Brasil, 2015).

\section{MATERIAIS E MÉTODOS}

Quanto à natureza dos resultados, este estudo caracteriza-se como pesquisa aplicada e assume a tipologia de Modelo Teórico-Conceitual, uma vez que visa promover uma dis- 
cussão da literatura, e, partindo-se das conclusões extraídas em união à análise dos dados coletados, desenvolver modelagens conceituais que resultem em uma nova teoria, corroborando para o avanço da ciência. Para o desenvolvimento do estudo, foram respeitadas uma sequência de atividades, descritas na Figura 1.

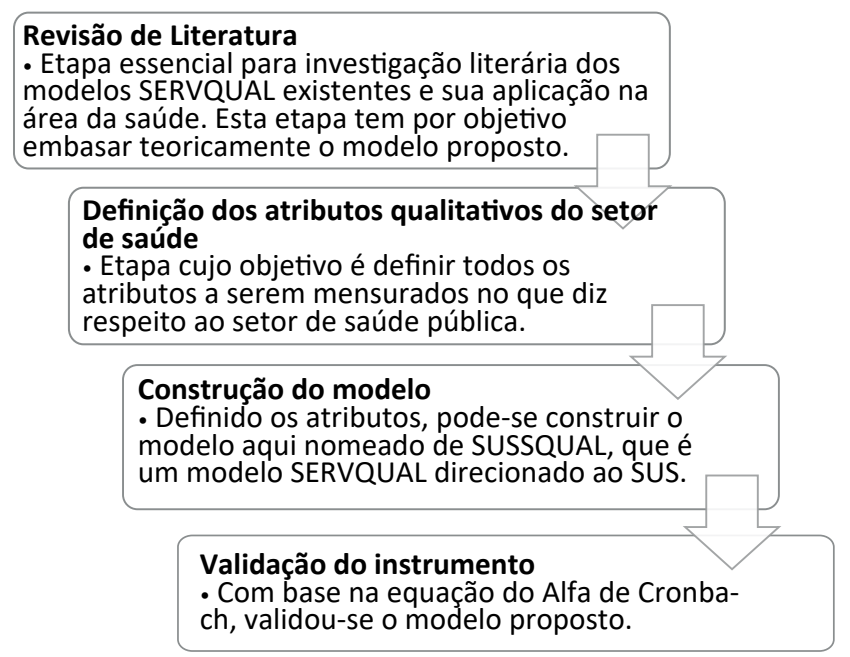

Figura 1. Etapas de pesquisa

O modelo a ser desenvolvido constitui-se como um instrumento de coleta, destinado a três públicos-alvo: usuários, profissionais e gestores de unidades de saúde pública municipal. Para verificar se o modelo proposto atendeu a seus objetivos, foi calculado o coeficiente alfa de Cronbach que, segundo Ganga (2012), deve ser submetido a três tipos de pessoas: colegas, especialistas da organização estudada e respondentes-alvo. O cálculo do coeficiente alfa é realizado segundo a Equação (1):

$$
\propto=\frac{k}{k-1} x\left[1-\frac{s_{i}^{2}}{s_{\text {soma }}^{2}}\right]
$$

Em que:

$$
S_{i}^{2}=\text { variância de cada variável k; }
$$

$S_{\text {soma }}^{2}=$ soma da variância de todas as variáveis.

O modelo proposto seguirá o método survey, funcionando como instrumento de coleta de variáveis categóricas ordinais, que podem ser definidas como variáveis qualitativas que apresentam ordenação entre as categorias, coletadas mediante utilização de escala do tipo Likert (Ganga, 2012). Neste estudo, no entanto, os instrumentos utilizados para coleta de dados serão constituídos de questões fechadas, com escalas do tipo Likert e de importância.
Para a construção do modelo, foi, inicialmente, realizada uma revisão literária com o objetivo de identificar variantes do modelo SERVQUAL e os critérios considerados pelo IOM, PNASS e legislação do SUS. Isso foi necessário para identificar aspectos qualitativos relevantes a serem considerados na avaliação de qualidade neste setor de serviços.

Após essa investigação, foram definidos os atributos qualitativos a serem considerados no modelo para avaliação da qualidade no setor da saúde pública. Isso permitiu a construção de dois questionários para aplicação do modelo proposto: a) usuários; b) profissionais de assistência e gestores.

\section{RESULTADOS}

O tópico que segue apresenta os resultados alcançados com o desenvolvimento do estudo até a apresentação do modelo proposto.

\section{SERVQUAL no setor de saúde}

Nesta secção serão abordadas utilizações do modelo SERVQUAL ao setor de saúde, presentes na literatura cientifica vigente. Para tal, fez-se necessário o refinamento das publicações a serem abordadas neste estudo, haja vista o grande número dessas produções disponíveis para acesso.

Para fins desse projeto, foram consideradas pertinentes as teses e dissertações publicadas no período compreendido de 2013 a 2016, originadas de cursos de Engenharia de Produção ofertados por instituições de ensino brasileiras, e que apresentassem a aplicação da ferramenta SERVQUAL a entidades prestadoras de serviços de saúde. A busca foi realizada no banco de teses e dissertações da Comissão de Aperfeiçoamento de Pessoal do Nível Superior (CAPES). 0 Quadro 1 apresenta um resumo das publicações selecionadas.

Quadro 1. Publicações sobre a aplicação do SERVQUAL em serviços de saúde

\begin{tabular}{|c|c|c|c|c|}
\hline $\begin{array}{c}\text { Tipolo- } \\
\text { gia }\end{array}$ & Autoria & Ano & Título & Abordagem \\
\hline Tese & $\begin{array}{c}\text { Guima- } \\
\text { rães } \\
\text { Júnior } \\
\text { DS }\end{array}$ & 2016 & $\begin{array}{c}\text { Modelo hierárquico e } \\
\text { multidimensional para } \\
\text { a mensuração da quali- } \\
\text { dade percebida no setor } \\
\text { de saúde suplementar }\end{array}$ & $\begin{array}{c}\text { Aplica } \\
\text { modelo } \\
\text { derivado do } \\
\text { SERVQUAL }\end{array}$ \\
\hline Tese & $\begin{array}{c}\text { Batista } \\
\text { DA }\end{array}$ & 2013 & $\begin{array}{c}\text { O uso da abordagem } \\
\text { Fuzzy para integração } \\
\text { das ferramentas QFD e } \\
\text { SERVQUAL em serviços } \\
\text { de saúde }\end{array}$ & $\begin{array}{c}\text { Propõe novo } \\
\text { modelo } \\
\text { integrado }\end{array}$ \\
\hline
\end{tabular}




\begin{tabular}{|c|c|c|c|c|}
\hline $\begin{array}{l}\text { Disserta- } \\
\text { ção }\end{array}$ & $\begin{array}{c}\text { Wegner } \\
\text { RS }\end{array}$ & 2016 & $\begin{array}{c}\text { Aplicação do SERVQUAL } \\
\text { e Analytic Hierarchy Pro- } \\
\text { cess: Ferramentas para } \\
\text { melhorias dos serviços } \\
\text { prestados pelo Hospital } \\
\text { Universitário de Santa } \\
\text { Maria/RS }\end{array}$ & $\begin{array}{c}\text { Aplica } \\
\text { modelo } \\
\text { de forma } \\
\text { integrada }\end{array}$ \\
\hline $\begin{array}{l}\text { Disserta- } \\
\text { ção }\end{array}$ & $\begin{array}{l}\text { Pereira } \\
\text { AMR }\end{array}$ & 2015 & $\begin{array}{l}\text { Avaliação da qualidade } \\
\text { em serviços públicos de } \\
\text { saúde em um hospital } \\
\text { utilizando a Análise } \\
\text { Hierárquica }\end{array}$ & $\begin{array}{l}\text { Aplica } \\
\text { modelo } \\
\text { derivado do } \\
\text { SERVQUAL } \\
\text { e propõe } \\
\text { modelo } \\
\text { adaptado }\end{array}$ \\
\hline $\begin{array}{l}\text { Disserta- } \\
\text { ção }\end{array}$ & $\begin{array}{l}\text { Queiroz } \\
\text { SFAM }\end{array}$ & 2014 & $\begin{array}{c}\text { Estudo da integração } \\
\text { do QFD com SERVQUAL } \\
\text { em hospitais privados } \\
\text { do Recife }\end{array}$ & $\begin{array}{l}\text { Propõe e } \\
\text { aplica nova } \\
\text { integração } \\
\text { do modelo }\end{array}$ \\
\hline $\begin{array}{l}\text { Disserta- } \\
\text { ção }\end{array}$ & $\begin{array}{l}\text { Maia } \\
\text { MCS }\end{array}$ & 2013 & $\begin{array}{l}\text { Uma abordagem para } \\
\text { avaliação da satisfa- } \\
\text { ção dos clientes em } \\
\text { empresas de serviços } \\
\text { de saúde: Aplicação da } \\
\text { integração dos modelos } \\
\text { SERVQUAL, KANO e QFD }\end{array}$ & $\begin{array}{l}\text { Propõe novo } \\
\text { modelo } \\
\text { integrado }\end{array}$ \\
\hline
\end{tabular}

Após uma investigação aos modelos SERVQUAL destinados à avaliação de saúde, fez-se necessário investigar os modelos de mensuração da qualidade que surgiram mediante variação do modelo SERVQUAL. No Quadro 2 é apresentado um resumo dos modelos mais usuais na literatura.

Quadro 2. Resumo das variações do modelo SERVQUAL

\begin{tabular}{|c|c|c|c|}
\hline Modelo & Ano & Autoria & Avaliação \\
\hline SERVPERF & 1992 & $\begin{array}{c}\text { Cronin \& } \\
\text { Taylor }\end{array}$ & Desempenho do serviço \\
\hline INTQUAL & 1997 & Caruana \& Pitt & Qualidade interna \\
\hline HOTELQUAL & 1999 & Delgado et al. & Qualidade da hotelaria \\
\hline HEdPERF & 2006 & Firdaus & $\begin{array}{c}\text { Qualidade do ensino } \\
\text { superior }\end{array}$ \\
\hline PERSPERF & 2009 & Yildiz \& Kara & $\begin{array}{c}\text { Qualidade do serviço no } \\
\text { ensino superior }\end{array}$ \\
\hline HOSPQUAL & 2011 & Rajaram & $\begin{array}{c}\text { Qualidade do serviço hos- } \\
\text { pitalar }\end{array}$ \\
\hline
\end{tabular}

O HOSPQUAL surgiu de um projeto de pesquisa para avaliação da qualidade do serviço de hospitais, com referência especial a distritos selecionados em Tamil Nadu, desenvolvido na Índia (Rajaram, 2011). Para mensuração da qualidade, o instrumento segue a lógica de análise estabelecida pelo SERVQUAL, ao coletar, mediante aplicação de questionários com escalas likert, as expectativas e percepções do público consumidor, e estabelecer os índices de qualidade das instituições. Porém, insere questionamentos específicos a setores hospitalares para apontamento dos usuários, relacionados com análise do comportamento dos médicos, dos profissionais da equipe de assistência, da qualidade administrativa dos hospitais e dos serviços prestados pelos hospitais.
Assim, o autor afirma que cada pergunta do SERVQUAL deveria ser reformulada para apresentação de maior consistência com a área do serviço particular a ser medida, além de ressaltar que o modelo referido não oferece um valor único atribuído à qualidade do serviço da organização como um todo, mas valores relacionados às cinco dimensões por ele abordadas, o que, segundo ele, fragilizaria o modelo SERVQUAL. O Quadro 3 apresenta a constituição da ferramenta HOSPQUAL.

Quadro 3. Dimensões do modelo HOSPQUAL.

\begin{tabular}{|c|c|}
\hline $\begin{array}{l}\text { DIMEN- } \\
\text { SÕES }\end{array}$ & HOSPQUAL - ITENS \\
\hline \multirow{7}{*}{$\begin{array}{l}\text { TANGIBILI- } \\
\text { DADE }\end{array}$} & $\begin{array}{l}\text { O hospital/clínica possui equipamentos de apa- } \\
\text { rência moderna. }\end{array}$ \\
\hline & $\begin{array}{l}\text { As instalações físicas do hospital são visualmente } \\
\text { atraentes. }\end{array}$ \\
\hline & $\begin{array}{l}\text { O pessoal do hospital/clínica possui aparência } \\
\text { limpa. }\end{array}$ \\
\hline & $\begin{array}{c}\text { Os materiais associados ao serviço (como pan- } \\
\text { fletos ou declarações) são visualmente atraentes } \\
\text { e demonstram qualidades de um excelente } \\
\text { hospital/clínica. }\end{array}$ \\
\hline & $\begin{array}{l}\text { Diversas atividades ao redor do hospital atendem } \\
\text { às necessidades dos clientes. }\end{array}$ \\
\hline & Localização conveniente. \\
\hline & $\begin{array}{l}\text { Os serviços são realizados em tempos adequa- } \\
\text { dos. }\end{array}$ \\
\hline \multirow{5}{*}{$\begin{array}{l}\text { CONFIABILI- } \\
\text { DADE }\end{array}$} & $\begin{array}{l}\text { O pessoal do hospital é confiável em atender às } \\
\text { requisições de serviço dos clientes. }\end{array}$ \\
\hline & $\begin{array}{l}\text { Os serviços são desempenhados no tempo } \\
\text { prometido. }\end{array}$ \\
\hline & $\begin{array}{l}\text { Os serviços são sempre executados de maneira } \\
\text { correta. }\end{array}$ \\
\hline & $\begin{array}{l}\text { As reivindicações dos pacientes são estabilizadas } \\
\text { sem atrasos desnecessários. }\end{array}$ \\
\hline & Exatidão dos relatórios médicos de despesas. \\
\hline \multirow{4}{*}{ RESPOSTA } & Facilidade de recepção no hospital. \\
\hline & $\begin{array}{l}\text { Prontidão de médicos, enfermeiros e pessoal } \\
\text { para prestação de serviços. }\end{array}$ \\
\hline & Resposta às necessidades dos clientes. \\
\hline & Facilidade de alta hospitalar. \\
\hline \multirow{5}{*}{ GARANTIA } & $\begin{array}{l}\text { Os médicos e enfermeiros que atuam no hospital } \\
\text { são competentes. }\end{array}$ \\
\hline & $\begin{array}{l}\text { Os médicos e enfermeiros que atuam no hospital } \\
\text { são experientes. }\end{array}$ \\
\hline & Respeito pela privacidade dos pacientes. \\
\hline & Atitude favorável em relação aos visitantes. \\
\hline & $\begin{array}{l}\text { Sentimentos de segurança e confiança são propi- } \\
\text { ciados pelo hospital. }\end{array}$ \\
\hline
\end{tabular}




\begin{tabular}{|c|c|}
\hline \multirow{5}{*}{ EMPATIA } & $\begin{array}{l}\text { Os serviços são prestados em tempo hábil aos } \\
\text { pacientes. }\end{array}$ \\
\hline & Há facilidade de comunicação com o médico. \\
\hline & $\begin{array}{c}\text { Obtém-se aprovação do paciente antes do teste e } \\
\text { do tratamento. }\end{array}$ \\
\hline & $\begin{array}{c}\text { Considera-se o problema dos pacientes, o que } \\
\text { almejam em seus corações. }\end{array}$ \\
\hline & Polidez do pessoal hospitalar. \\
\hline
\end{tabular}

Além disso, dentre os modelos existentes direcionados à avaliação da qualidade em serviços, o HOSPQUAL desenvolvido por Rajaram (2011), embora seja voltado para o setor hospitalar, trata-se apenas de mais uma aplicação adaptada da ferramenta SERVQUAL, na qual o autor personaliza as questões que constituem os instrumentos do modelo referido. O Quadro 4 apresenta as dimensões do SERVQUAL, HOSPQUAL e as propostas pelo modelo deste estudo - o SUSSQUAL.

Quadro 4. Comparação das dimensões SERVQUAL, HOSPQUAL e SUSSQUAL

\begin{tabular}{|c|c|c|c|}
\hline \multirow{9}{*}{ 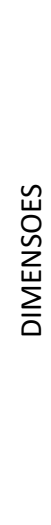 } & SERVQUAL & HOSPQUAL & SUSSQUAL \\
\hline & Tangibilidade & Tangibilidade & Tangibilidade \\
\hline & Confiabilidade & Confiabilidade & Acesso \\
\hline & Presteza & Resposta & $\begin{array}{l}\text { Centralidade no } \\
\text { paciente }\end{array}$ \\
\hline & Segurança & Garantia & Segurança \\
\hline & Empatia & Empatia & Empatia \\
\hline & - & - & Eficiência \\
\hline & - & - & $\begin{array}{c}\text { Eficácia e efetivi- } \\
\text { dade }\end{array}$ \\
\hline & - & - & Legislação \\
\hline
\end{tabular}

O modelo HOSPQUAL é uma adaptação do SERVQUAL, não havendo, portanto, acréscimos ou modificações de dimensões qualitativas. O modelo SUSSQUAL, por sua vez, apresenta alguns acréscimos de informações. Este modelo foi elaborado com o intuito de viabilizar a medição dos níveis qualitativos das dimensões da qualidade dos serviços prestados pelo SUS à população, de forma mais coerente com a natureza do serviço em questão.

O método SUSSQUAL agrega dimensões qualitativas que foram desconsideradas na formulação do modelo SERVQUAL, próprias da área da saúde, reverenciadas pelo IOM, responsável pela formulação de estratégias qualitativas em serviços de saúde internacionais e pelo Programa PNASS, política responsável pela auditoria dos serviços públicos de saúde do Brasil, além de considerar aspectos legislativos constitucionais. Para a proposta do modelo, foram considerados aspectos do IOM e PNASS. O Quadro 5 apresenta a relação entre as dimensões qualitativas entre eles.

Quadro 5. Relação entre as dimensões qualitativas do PNASS, IOM, SERVQUAL E SUSSQUAL.

\begin{tabular}{|c|c|c|c|c|}
\hline \multirow{9}{*}{ 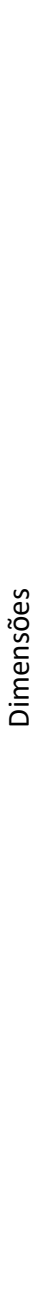 } & PNASS & IOM & $\begin{array}{c}\text { SER- } \\
\text { VQUAL }\end{array}$ & $\begin{array}{l}\text { SUSS- } \\
\text { QUAL }\end{array}$ \\
\hline & $\begin{array}{c}\text { Bloco II - Apoio Técnico e } \\
\text { Logístico para a Produção } \\
\text { do Cuidado - Critério } 7 \text { - } \\
\text { Gestão da Infraestrutura e } \\
\text { Ambiência }\end{array}$ & - & $\begin{array}{l}\text { Tangibi- } \\
\text { lidade }\end{array}$ & $\begin{array}{c}\text { Tangibili- } \\
\text { dade }\end{array}$ \\
\hline & $\begin{array}{l}\text { Questionário Dirigido aos } \\
\text { Usuários - Item } 1 \text { - Agilida- } \\
\text { de no Agendamento do } \\
\text { Atendimento - Item } 2 \text { - } \\
\text { Agilidade no Atendimento }\end{array}$ & $\begin{array}{c}\text { Eficiên- } \\
\text { cia }\end{array}$ & - & Eficiência \\
\hline & - & $\begin{array}{l}\text { Efetivi- } \\
\text { dade }\end{array}$ & - & $\begin{array}{l}\text { Eficácia e } \\
\text { efetivida- } \\
\text { de }\end{array}$ \\
\hline & $\begin{array}{l}\text { Questionário Dirigido aos } \\
\text { Usuários - Item } 3 \text { - Acolhi- } \\
\text { mento - Item } 8 \text { - Marcas } \\
\text { de Humanização }\end{array}$ & $\begin{array}{l}\text { Cen- } \\
\text { tralida- } \\
\text { de no } \\
\text { Pacien- } \\
\text { te }\end{array}$ & $\begin{array}{c}\text { Preste- } \\
\text { za }\end{array}$ & $\begin{array}{l}\text { Centrali- } \\
\text { dade no } \\
\text { paciente }\end{array}$ \\
\hline & $\begin{array}{l}\text { Bloco II - Apoio Técnico e } \\
\text { Logístico para a Produção } \\
\text { do Cuidado - Critério } 6 \text { - } \\
\text { Gerenciamento de risco e } \\
\text { segurança do paciente }\end{array}$ & $\begin{array}{l}\text { Segu- } \\
\text { rança }\end{array}$ & $\begin{array}{l}\text { Segu- } \\
\text { rança }\end{array}$ & $\begin{array}{c}\text { Seguran- } \\
\text { ça }\end{array}$ \\
\hline & - & - & $\begin{array}{c}\text { Empa- } \\
\text { tia }\end{array}$ & Empatia \\
\hline & $\begin{array}{l}\text { Questionário Dirigido aos } \\
\text { Usuários - Item } 4 \text { - Con- } \\
\text { fiança }\end{array}$ & Acesso & $\begin{array}{l}\text { Confia- } \\
\text { bilidade }\end{array}$ & Acesso \\
\hline & - & $\begin{array}{l}\text { Equi- } \\
\text { dade }\end{array}$ & - & $\begin{array}{l}\text { Legisla- } \\
\text { ção }\end{array}$ \\
\hline
\end{tabular}

Assim, com base nos conceitos do SERVQUAL, IOM, PNASS e a legislação regulamentadora, as dimensões do SUSSQUAL definidas são: tangibilidade, eficiência, eficácia, efetividade, centralidade no paciente, segurança, empatia, acesso e legislação.

\section{Proposta do modelo}

Com base nas referências apresentadas, o modelo SUSSQUAL surge com o intuito de atuar como ferramenta auxiliar para avaliação da qualidade do serviço prestado pelos estabelecimentos e/ou sistemas de saúde subsidiados pelo SUS. 
Para prosseguir na delimitação do objeto a ser avaliado é necessário definir quais os níveis da realidade que se quer estudar. O campo da saúde é formado por instituições públicas e privadas que desenvolvem ações de promoção, prevenção e cura voltadas para a população. O SUS apresenta suas particularidades, sendo um sistema relativamente novo no País, além de ser constitucional. Esse fator justifica a necessidade de avaliação do serviço, considerando os diversos aspectos do sistema e apontamento de diversos agentes envolvidos na dinâmica da prestação de serviços. Assim, o SUSSQUAL considera novas dimensões e uma abordagem específica com base no contexto brasileiro.

O modelo proposto é constituído por dois instrumentos de coleta. O primeiro é voltado para os usuários e profissionais atuantes na assistência médica, da área da saúde pública. E o segundo, de caráter opcional e complementar, é direcionado aos gestores de cada instituição pública de saúde.

Os usuários dos serviços, representantes da população, os profissionais da saúde e gestores dos serviços têm objetivos e percepções distintas em relação aos serviços de saúde, e, geralmente, dão prioridade a aspectos diferentes quando avaliam a qualidade das ações de saúde. Assim, é preciso democratizar as avaliações que pretendem influenciar processos de tomada de decisão nos serviços, considerando que o envolvimento dos diversos atores amplia o poder da avaliação de desvendar distintos aspectos de uma mesma intervenção e de seus efeitos (Serapione, 1999, apud Hartz; Silva, 2005, p. 59).

O modelo SUSSQUAL apresenta a constituição de cada instrumento dividida em três seções: questões voltadas à estratificação do público respondente; itens de verificação qualitativa das diversas dimensões que constituem o processo de prestação de serviços; e, por fim, a seção que solicita o grau de importância de cada dimensão qualitativa sob a perspectiva do respondente.

$\mathrm{O}$ instrumento proposto, no entanto, considera apenas a assimilação da percepção dos aspectos de qualidade explorados, não solicitando a expectativa dos públicos em relação ao serviço. Tal fato justifica-se considerando que a qualidade pode ser definida como a "habilidade das características inerentes de um produto, sistema ou processo de atender as necessidades dos clientes e outras partes interessadas" (ISO/DIS 9000:2000, 1999). Ao estipular-se a avaliação da percepção do usuário em uma escala de 1 a 5, considera-se que o apontamento 5 expressa que determinado aspecto conseguiu satisfazer por completo ao respondente, mediante comparativo natural entre o que este compreende como nível de qualidade desejável em determinado aspecto e o nível de qualidade do qual usufruiu. O nível de percepção da qualidade em si já aponta o grau qualitativo, fator que torna desnecessária a solicitação de expectativa do respondente.
Assim, a ferramenta proposta neste estudo se posiciona como meio propício à identificação dos níveis de serviços de saúde ofertados, e ao aprimoramento contínuo das atribuições que os constituem, além de funcionar como instrumento de controle de desempenho para a organização que dela valer-se.

O modelo é constituído por dois questionários (usuários e gestores) relacionados ao sistema público de saúde. O primeiro (Apêndice 2), destinado aos usuários, é composto por 21 questões subdivididas nas nove dimensões propostas. Para os gestores (Apêndice 3), são 29 itens para avaliação das nove dimensões propostas pelo modelo. $O$ modelo ainda conta com um levantamento inicial do respondente (Apêndice 1), servindo como início para ambos os questionários. A Tabela 1 apresenta a quantidade de questões propostas em cada dimensão dos questionários.

Tabela 1. Quantidade de questões dos questionários

\begin{tabular}{|c|c|c|c|}
\hline $\begin{array}{c}\text { Gru- } \\
\text { po }\end{array}$ & $\begin{array}{c}\text { Dimensões } \\
\text { qualitativas }\end{array}$ & $\begin{array}{c}\text { Questões - } \\
\text { Usuários }\end{array}$ & $\begin{array}{c}\text { Questões - } \\
\text { Gestores }\end{array}$ \\
\hline 1 & Tangibilidade & 3 & 7 \\
\hline 2 & Eficiência & 3 & 4 \\
\hline 3 & Eficácia & 1 & 1 \\
\hline 4 & Efetividade & 1 & 1 \\
\hline 5 & $\begin{array}{c}\text { Centralidade no } \\
\text { Paciente }\end{array}$ & 2 & 1 \\
\hline 6 & Segurança & 2 & 2 \\
\hline 7 & Empatia & 2 & 4 \\
\hline 8 & Acesso & 2 & 2 \\
\hline 9 & Legislação & 5 & 7 \\
\hline
\end{tabular}

Os itens que constituem a ferramenta são afirmativas relacionadas aos serviços ofertados pela unidade de saúde em avaliação, para as quais o respondente deve indicar seu grau de concordância em uma escala likert de 5 pontos, em que 1 expressa discordância total e 5 concordância completa à afirmativa. Além disso, a ferramenta solicita o grau de importância de determinada dimensão qualitativa para a produção de um serviço considerado ótimo em saúde. Nesta seção, o respondente deve indicar o quanto considera determinado aspecto relevante para a propiciação de um serviço considerado qualitativo, em uma escala de importância de 5 pontos, em que 1 expressa um critério sem importância, 2 indica um critério com pouca importância, 3 informa um critério indiferente quanto à sua relevância, 4 expressa um critério de moderada importância e 5 indica um critério de extrema importante.

A utilização desse instrumento é importante para o respaldo e análise dos resultados obtidos na aplicação do instrumento "Questionário usuários/profissionais de assistên- 
cia", haja vista que tem como objetivo direcionar o olhar do avaliador sobre as possíveis causas dos gaps qualitativos apontados pelos usuários e profissionais de assistência.

\section{Validação do instrumento}

Para a validação do constructo utilizou-se a análise estatística por meio da aplicação do alfa de Cronbach, a fim da verificação da taxa de confiabilidade dos instrumentos desenvolvidos para constituição do modelo proposto.

Para tal, são estimados quantitativos mínimos de questionários respondidos pelos três públicos do modelo proposto neste estudo, a serem analisados estatisticamente. Assim, um valor igual ou superior ao estabelecido é utilizado para análise do modelo. São utilizados questionários provenientes da aplicação em quatro tipologias de estabelecimentos de saúde, sendo dois centros de saúde (US-A e US-E), uma clínica especializada (US-D), um hospital geral (US-C) e um hospital especializado (US-B). Em cada estabelecimento foram entrevistados 40 usuários, 20 profissionais e um gestor. A Tabela 2 apresenta os resultados do coeficiente para cada unidade investigada e um coeficiente geral para os 200 usuários e 100 profissionais entrevistados.

Tabela 2. Alfa de Cronbach por unidade de saúde.

\begin{tabular}{|c|c|c|c|c|c|c|}
\hline Amostra & US (A) & US (B) & US (C) & US (D) & US (E) & Total \\
\hline Usuários & 0,930 & 0,907 & 0,929 & 0,920 & 0,943 & 0,922 \\
\hline $\begin{array}{c}\text { Profissio- } \\
\text { nais }\end{array}$ & 0,794 & 0,883 & 0,624 & 0,647 & 0,858 & 0,858 \\
\hline
\end{tabular}

De acordo com a classificação estabelecida por Freitas e Rodrigues (2005), os indicadores ficaram entre moderado, alto e muito alto. Para esses indicadores, os níveis estão consistentes para o objetivo que o questionário se propõe. Para os gestores foi feito uma análise geral dos cinco entrevistados, obtendo-se um coeficiente de 0,971 , apresentando consistência muito alta.

\section{CONSIDERAÇÕES FINAIS}

Este trabalho teve como objetivo o desenvolvimento de um modelo que melhor mensurasse a qualidade do serviço prestado pelas instituições públicas de saúde brasileiras. A união de conceitos abordados no IOM, PNASS e SERVQUAL serviu de base para a formulação do modelo proposto e, assim, mediante a aplicação, foi possível coletar dados pertinentes à identificação dos critérios qualitativos a serem priorizados para melhoria do sistema estudado.
A revisão da literatura foi realizada com intuito de embasar teoricamente o estudo. Foram investigados os modelos SERVQUAL para área da saúde e as variações existentes desta ferramenta para construir o modelo proposto. Os conceitos de IOM, PNASS e legislação do SUS foram integrados e atuaram como norteadores na elaboração dos instrumentos de coleta, a fim de que ele pudesse abordar os itens e dimensões que mais se aproximassem da realidade dos usuários e profissionais da saúde.

O modelo pode ser aplicado à realidade do SUS, sendo que pode-se levantar a realidade local e direcionar melhorias de forma mais eficaz. O modelo se mostrou robusto e valido após ser validado por meio do coeficiente alfa de Cronbach. Sendo assim, o modelo é consistente aos objetivos propostos. Como proposta de estudo futuro, sugere-se uma aplicação do modelo após validado para uma análise pertinente dos dados levantados.

\section{REFERÊNCIAS}

Allen Jr., L.V. 2016. Introdução à farmácia de Remington. University of Sciences in Philadelphia. Porto Alegre: Artmed.

Batalha, M.O. (Org.).2008. Introdução à Engenharia de Produção. Rio de Janeiro: Elsevier.

Batista, D.A. 2013. O uso da Abordagem Fuzzy para a integração das ferramentas QFD e SERVQUAL em serviços de saúde [tese]. Recife: Universidade Federal de Pernambuco.

Brasil. Ministério da Saúde. Secretaria-Executiva. Departamento de Regulação, Avaliação e Controle de Sistemas. 2015. PNASS: Programa Nacional de Avaliação dos Serviços de Saúde. Brasília: Ministério da Saúde.

Caruana, A. and Pitt, L. 1997. INTQUAL-An Internal Measure of Service Quality and the Link between Service Quality and Business Performance. European Journal of Marketing 31, 604-616. https://doi.org/10.1108/03090569710176600

Conselho Federal de Farmácia - CFF. 2015. Brasil é um dos países que menos investem em saúde pública. https://www.cff. org.br/noticia. .php?id=2937\&titulo=Brasil+\%C3\%A9+um+dos + pa\%C3\%ADses+que+menos+investem+em+sa\%C3\%BAde+p \%C3\%BAblica

Cronin, J.J., \& Taylor, S.A. 1992. Measuring service quality: A reexamination and extension. Journal of Marketing 56, 3:5568. https://doi.org/10.2307/1252296

Delgado, C.F. et al. 1999. Hotelqual: Una escala para medir calidad percebida em servicios de alojamento. Estudios Turisticos, 139:93-108.

Ferreira, S.P.C. 2015. Modelo de satisfação de clientes do Hospital das Forças Armadas. Relatório Científico do Trabalho de Investigação Aplicada. Lisboa. 
Firdaus, A. 2006. The development of HEdPERF: a new measuring instrument of service quality for the higher education sector. International Journal of Consumer Studies 30, 6:569581.

Freitas, A.L.P.; Rodrigues, S.G. 2005. Avaliação da Confiabilidade de Questionários: uma Análise Utilizando o Coeficiente de Alpha de Cronbach. Anais do XII SIMPEP - Simpósio de Engenharia de Produção, Bauru, SP, Brasil, 7 a 9 de novembro de 2005.

Ganga, G.M.D. 2012. Trabalho de Conclusão de Curso (TCC) na engenharia de produção: um guia prático de conteúdo e forma. São Paulo: Atlas.

Guimarães Júnior, D.S. 2016. Modelo Hierarquico e Multidimensional para mensuração da qualidade percebida no setor de saúde suplementar [tese]. Recife: Universidade Federal de Pernambuco.

Hartz, Z. M. D. A., \& Silva, L. M. V. D. 2005. Avaliação em saúde: dos modelos teóricos à prática na avaliação de programas e sistemas de saúde. Rio de Janeiro: Fiocruz.

Institute of Medicine. 1990. Crossing the quality chasm: a new health system for the 21st century. Washington DC: National Academy Press.

Instituto Trata Brasil, Instituto Brasileiro de Opinião Pública e Estatística. 2012. A percepção da população quanto ao Saneamento Básico e a responsabilidade do Poder Público. São Paulo: Instituto Trata Brasil, Ibope.

ISO/DIS 9000:2000, Projeto de Norma - Quality Management Systems - fundamentals and vocabulary - ISSO, proposta datada de 25/11/1999.

Maia, M.C.S. 2013. Uma abordagem para avaliação da satisfação dos clientes em empresas de serviços de saúde: Aplicação da integração dos modelos SERVQUAL, KANO e QFD [dissertação]. Recife: Universidade Federal de Pernambuco.
Mendes, E.V. 2002. Sistema Nacional de Saúde no Brasil - SUS e Sistema Complementar. Ano III, no1.

Mezomo, J.C. 2001. Gestão da Qualidade na Saúde: Princípios Básicos. São Paulo: Manole.

Parasuraman, A. et al. 1988. SERVQUAL: A Multiple-Item Scale for Measuring Consumer Perceptions of Service Quality. Journal of Retailing 4, 1.

Pereira, A.M.R. 2015. Avaliação da qualidade em serviços públicos de saúde em um hospital usando a análise hierárquica [dissertação]. Recife: Universidade Federal de Pernambuco.

Prates, M. 2012. 5 países onde a saúde pública funciona. $\mathrm{Pu}$ blicado 13 jun. 2012. http://exame.abril.com.br/mundo/5-paises-onde-a-saude-publica-funciona/

Queiroz, S.F.A.M. 2014. Estudo da integração do QFD com SERVQUAL em hospitais privados do Recife [dissertação]. Recife: Universidade Federal de Pernambuco.

Rajaram, S. 2011. Service Quality Assessment in Hospitals with special reference to Selected Districts in Tamil Nadu. Indian Institute of Public Administration.

Sousa, T.C.G. et al. 2011. Comparação de modelos de qualidade de serviços: proposição estratégica para instituições de ensino superior. Seminários em Administração, SemeAd, out.

Vecina Neto, G.; Malik, A.M. 2014. Gestão em Saúde. Rio de Janeiro: Guanabara Koogan.

Wegner, R.S. 2016. Aplicação do SERVQUAL e Analytic Hierarchy Process: Ferramentas para melhoria dos serviços prestados pelo Hospital Universitário de Santa Maria, RS [dissertação]. Rio Grande do Sul: Universidade Federal de Santa Maria.

Yildiz, S.M.; Kara, A. 2009. The PESPERF scale: An instrument for measuring service quality in the School of Physical Education and Sports Sciences (PESS). Quality Assurance in Education 17, 4:393-415. 
Apêndice 1 - Caracterização dos usuários - Questionário "usuários/profissionais de assistência"

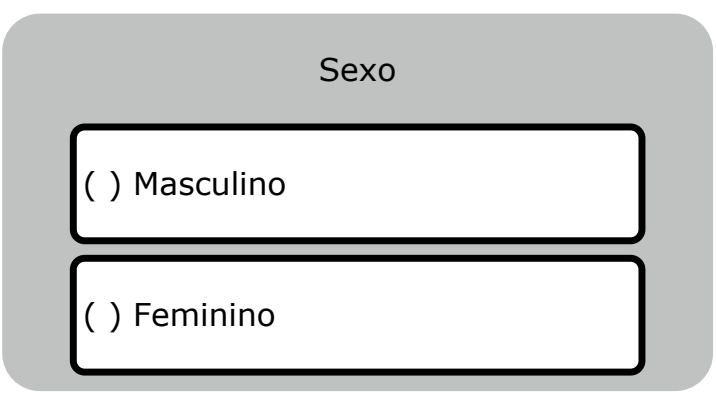

Faixa Etária

(C) Até19 anos

(1) De 20 à 29 anos

(1) De 30 a 39 anos

(C) De 40 a 49 anos

(C)De 50 a 59 anos

(1) 60 anos ou mais

Renda Familiar

( ) De $\mathrm{R} \$ 600,00$ a $\mathrm{R} \$ 880,00$

( ) De $R \$ 881,00$ a $R \$ 1000,00$

( ) De $R \$ 1001,00$ a $R \$ 1500,00$

( ) De $R \$ 1501,00$ a $R \$ 2000,00$

( ) De $R \$ 2001,00$ a $R \$ 3000,00$

( ) Acima $\mathrm{R} \$ 3000,00$

( ) Sem renda familiar fixa

\section{Estado Civil}

( ) Solteiro

( ) Casado

( ) Divorciado

( ) Viúvo

( ) União Estável

\section{Escolaridade}

(C) Fundamental incompleto

(1) Fundamental Completo

() Ensino Médio Incompleto

(1) Ensino Médio Completo

(C) Ensino Superior Incompleto

(c) Ensino Superior Completo

Quantas vezes você já utilizou esta Unidade de Atendimento para realizar algum procedimento?

\section{( ) Apenas $1 \mathrm{vez}$}

( ) De 2 a 5 vezes

( ) De 6 a 9 vezes

( ) De 10 a 19 vezes

( ) 20 a 29 vezes

( ) Acima de 30 vezes 
Apêndice 2 - Questionário "usuários / profissionais de assistência"

Instruções: As frases a seguir referem-se à sua percepção sobre a prestação de serviço desta unidade de atendimento de saúde (posto ou hospital) assistida pelo SUS (Sistema Único de Saúde). Para cada uma das afirmações indique o quanto você acredita que a unidade de atendimento atinge na escala de 1 a 5 .

\begin{tabular}{|c|c|c|c|c|c|c|c|}
\hline \multirow{3}{*}{ Tangibilidade } & 1 & $\begin{array}{l}\text { A unidade de saúde possui equipamentos adequados e em condi- } \\
\text { ções de uso para exercício satisfatório de suas atividades. }\end{array}$ & 1 & 2 & 3 & 4 & 5 \\
\hline & 2 & $\begin{array}{c}\text { As instalações encontram-se limpas, com sinalização norteadora e } \\
\text { climatização adequada. }\end{array}$ & 1 & 2 & 3 & 4 & 5 \\
\hline & 3 & $\begin{array}{l}\text { A equipe de assistência médica utiliza trajes e acessórios (luvas, más- } \\
\text { caras etc.) em condições adequadas para atendimento. }\end{array}$ & 1 & 2 & 3 & 4 & 5 \\
\hline \multirow{3}{*}{ Eficiência } & 4 & $\begin{array}{l}\text { Os procedimentos são agendados em tempo hábil às necessidades } \\
\text { dos usuários. }\end{array}$ & 1 & 2 & 3 & 4 & 5 \\
\hline & 5 & $\begin{array}{l}\text { No dia do procedimento, o serviço é prestado no horário marcado } \\
\text { (consultas, cirurgias) e/ou entregue no prazo estabelecido (exames). }\end{array}$ & 1 & 2 & 3 & 4 & 5 \\
\hline & 6 & $\begin{array}{l}\text { A resolução de pequenos procedimentos ocorre de maneira prática } \\
\text { (curativos, liberação de medicamentos etc.). }\end{array}$ & 1 & 2 & 3 & 4 & 5 \\
\hline Eficácia & 7 & O serviço é executado de forma correta na primeira vez. & 1 & 2 & 3 & 4 & 5 \\
\hline Efetividade & 8 & Os procedimentos têm resultados de qualidade ao final do serviço. & 1 & 2 & 3 & 4 & 5 \\
\hline \multirow{2}{*}{$\begin{array}{l}\text { Centralidade } \\
\text { no Paciente }\end{array}$} & 9 & $\begin{array}{l}\text { A equipe administrativa mantém os usuários informados com exati- } \\
\text { dão acerca dos procedimentos a serem realizados. }\end{array}$ & 1 & 2 & 3 & 4 & 5 \\
\hline & 10 & $\begin{array}{l}\text { A equipe de assistência médica procura ajudar sempre que necessá- } \\
\text { rio aos pacientes e familiares no esclarecimento de dúvidas quanto } \\
\text { ao estado de saúde do usuário. }\end{array}$ & 1 & 2 & 3 & 4 & 5 \\
\hline \multirow[b]{2}{*}{ Segurança } & 11 & $\begin{array}{l}\text { Os usuários sentem-se seguros ao utilizar os serviços de saúde públi- } \\
\qquad \text { cos da unidade de saúde. }\end{array}$ & 1 & 2 & 3 & 4 & 5 \\
\hline & 12 & $\begin{array}{c}\text { A equipe administrativa e a equipe de assistência média possuem } \\
\text { conhecimento adequado para responder aos questionamentos dos } \\
\text { usuários, quando solicitados. }\end{array}$ & 1 & 2 & 3 & 4 & 5 \\
\hline \multirow[t]{2}{*}{ Empatia } & 13 & $\begin{array}{l}\text { A equipe de assistência médica busca compreender a necessidade de } \\
\text { cada paciente, e a partir daí, desenvolve atendimento personalizado, } \\
\text { que atenda às demandas dos usuários da melhor forma possível. }\end{array}$ & 1 & 2 & 3 & 4 & 5 \\
\hline & 14 & $\begin{array}{l}\text { A equipe administrativa dá atenção aos pacientes e familiares, atra- } \\
\text { vés de apoio moral e esclarecimento de dúvidas. }\end{array}$ & 1 & 2 & 3 & 4 & 5 \\
\hline \multirow[b]{2}{*}{ Acesso } & 15 & $\begin{array}{l}\text { A unidade de saúde realiza todos os procedimentos para que o tem- } \\
\text { po de espera seja reduzido. }\end{array}$ & 1 & 2 & 3 & 4 & 5 \\
\hline & 16 & $\begin{array}{c}\text { Há agilidade no atendimento de todos os setores da unidade de } \\
\text { saúde que têm a interface urgência/emergência e/ou atendimento } \\
\text { prioritário. }\end{array}$ & 1 & 2 & 3 & 4 & 5 \\
\hline \multirow{5}{*}{ Legislação } & 17 & $\begin{array}{l}\text { Não há descriminação de qualquer natureza para efetivação de aten- } \\
\text { dimento e/ou agendamento para utilização dos serviços de saúde } \\
\text { ofertados pela unidade. }\end{array}$ & 1 & 2 & 3 & 4 & 5 \\
\hline & 18 & $\begin{array}{l}\text { Há igualdade na assistência à saúde. Assim, os usuários são atendi- } \\
\text { dos sem preconceitos ou privilégios de qualquer natureza, salvo as } \\
\text { prioridades estabelecidas em lei. }\end{array}$ & 1 & 2 & 3 & 4 & 5 \\
\hline & 19 & São oferecidos serviços preventivos e curativos em caráter integral. & 1 & 2 & 3 & 4 & 5 \\
\hline & 20 & $\begin{array}{l}\text { A autonomia dos pacientes e acompanhantes é respeitada e estimu- } \\
\text { lada. }\end{array}$ & 1 & 2 & 3 & 4 & 5 \\
\hline & 21 & $\begin{array}{l}\text { A unidade de atendimento se atenta para divulgação quanto aos } \\
\text { tipos de serviços de saúde ofertados na instituição. }\end{array}$ & 1 & 2 & 3 & 4 & 5 \\
\hline
\end{tabular}




\begin{tabular}{|c|c|c|c|c|c|c|c|}
\hline \multicolumn{8}{|c|}{ Importância de cada dimensão dentro do setor público de saúde } \\
\hline Tangibilidade & 1 & $\begin{array}{l}\text { Característica do que é tangível, palpável, que pode ser tocado (es- } \\
\text { trutura do local, objetos etc.). }\end{array}$ & 1 & 2 & 3 & 4 & 5 \\
\hline Eficiência & 2 & $\begin{array}{l}\text { Realizar os procedimentos com o mínimo de perda, por meio do me- } \\
\text { Ihor uso possível do tempo, dos materiais e dos recursos humanos. }\end{array}$ & 1 & 2 & 3 & 4 & 5 \\
\hline Eficácia & 3 & Realizar os procedimentos da maneira correta. & 1 & 2 & 3 & 4 & 5 \\
\hline Efetividade & 4 & Realizar os procedimentos de maneira certa e com qualidade & 1 & 2 & 3 & 4 & 5 \\
\hline $\begin{array}{l}\text { Centralidade } \\
\text { no Paciente }\end{array}$ & 5 & $\begin{array}{l}\text { Parceria entre profissionais e pacientes a fim de garantir que o cuida- } \\
\text { do respeite as necessidades e preferências dos pacientes. }\end{array}$ & 1 & 2 & 3 & 4 & 5 \\
\hline Segurança & 6 & $\begin{array}{l}\text { A prestação de serviço transmite a segurança de que o procedimento } \\
\text { será realizado sem danos aos pacientes. }\end{array}$ & 1 & 2 & 3 & 4 & 5 \\
\hline Empatia & 7 & $\begin{array}{l}\text { Ajudar e buscar compreender as necessidades do paciente, se colo- } \\
\text { cando no lugar dele e agindo segundo princípios morais. }\end{array}$ & 1 & 2 & 3 & 4 & 5 \\
\hline Acesso & 8 & $\begin{array}{l}\text { Tempo de espera e agilidade no atendimento em todos os setores da } \\
\text { unidade. }\end{array}$ & 1 & 2 & 3 & 4 & 5 \\
\hline Legislação & 9 & $\begin{array}{l}\text { Conformidade com a lei, igualdade, imparcialidade, moderação, } \\
\text { justiça, equivalência. }\end{array}$ & 1 & 2 & 3 & 4 & 5 \\
\hline $\begin{array}{l}\text { Equipe de } \\
\text { Assistência } \\
\text { Médica }\end{array}$ & \multicolumn{7}{|c|}{$\begin{array}{l}\text { Médico, enfermeiro, técnico de enfermagem, assistente social, psicólogo, farmacêutico e demais profissionais envol- } \\
\text { vidos diretamente com a prestação de serviços de atenção à saúde aos usuários. }\end{array}$} \\
\hline $\begin{array}{l}\text { Equipe Admi- } \\
\text { nistrativa }\end{array}$ & \multicolumn{7}{|c|}{$\begin{array}{l}\text { Recepcionista, atendente, auxiliar administrativo e demais profissionais que atuem em serviços de apoio administra- } \\
\text { tivo. }\end{array}$} \\
\hline
\end{tabular}

Apêndice 3-Questionário "gestores"

\begin{tabular}{|c|c|c|c|c|c|c|c|}
\hline \multicolumn{3}{|c|}{$\begin{array}{l}\text { Obs.: As afirmações a seguir referem-se à avaliação da unidade de saúde. para cada afirmativa, } \\
\text { indique o quanto determinada afirmação é condizente com a realidade da unidade avaliada. } \\
\text { Considere: } 1 \text { - discordo totalmente e } 5 \text { - concordo totalmente }\end{array}$} & \multirow{2}{*}{ 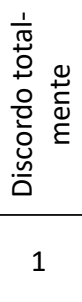 } & \multirow{2}{*}{ 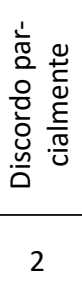 } & \multirow{2}{*}{ 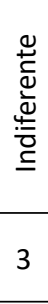 } & \multirow{2}{*}{ 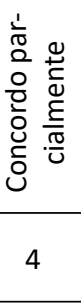 } & \multirow{2}{*}{ 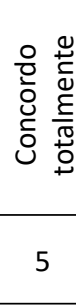 } \\
\hline \multirow{7}{*}{$\begin{array}{l}\text { Tangibi- } \\
\text { lidade }\end{array}$} & $1 \mathrm{a}$ & $\begin{array}{l}\text { A unidade dispõe de equipamentos médico-hospitalares em condições } \\
\text { adequadas para exercício satisfatório de suas atividades. }\end{array}$ & & & & & \\
\hline & $1 b$ & São realizados procedimentos de manutenção periódica nos equipamentos. & 1 & 2 & 3 & 4 & 5 \\
\hline & $2 a$ & $\begin{array}{l}\text { A unidade dispõe de materiais de limpeza em quantidade e condições } \\
\text { satisfatórias para uso. }\end{array}$ & 1 & 2 & 3 & 4 & 5 \\
\hline & $2 b$ & $\begin{array}{l}\text { A unidade dispõe de sinalização auxiliadora e identificação departamental } \\
\text { para auxílio e promoção da autonomia dos usuários. }\end{array}$ & 1 & 2 & 3 & 4 & 5 \\
\hline & 2c & $\begin{array}{l}\text { A climatização predial é adequada, proporcionando bem-estar aos profis- } \\
\text { sionais e usuários. }\end{array}$ & 1 & 2 & 3 & 4 & 5 \\
\hline & $3 a$ & $\begin{array}{l}\text { A equipe de assistência médica é orientada à utilização de trajes adequa- } \\
\text { dos ao ambiente assistencial. }\end{array}$ & 1 & 2 & 3 & 4 & 5 \\
\hline & $3 b$ & $\begin{array}{c}\text { A equipe de assistência médica utiliza materiais de apoio em quantitativo } \\
\text { e condições de uso adequadas para atendimento dos usuários (equipa- } \\
\text { mentos de proteção individual). }\end{array}$ & 1 & 2 & 3 & 4 & 5 \\
\hline
\end{tabular}




\begin{tabular}{|c|c|c|c|c|c|c|c|}
\hline \multirow{4}{*}{$\begin{array}{l}\text { Eficiên- } \\
\text { cia }\end{array}$} & $4 a$ & A unidade utiliza-se de agendamento para atendimento ordenado dos usuários. & 1 & 2 & 3 & 4 & 5 \\
\hline & $4 b$ & $\begin{array}{l}\text { A unidade não recebe reclamações frequentes dos usuários acerca da } \\
\text { distância temporal entre a solicitação de agendamentos e a execução do } \\
\text { serviço requerido. }\end{array}$ & 1 & 2 & 3 & 4 & 5 \\
\hline & $5 a$ & $\begin{array}{l}\text { No dia da prestação do serviço requerido pelo usuário, o procedimento é } \\
\text { executado no horário marcado (ex.: consultas, procedimentos cirúrgicos etc.) } \\
\text { e/ou entregue no prazo estabelecido (ex.: entrega de resultados de exames). }\end{array}$ & 1 & 2 & 3 & 4 & 5 \\
\hline & $6 a$ & $\begin{array}{l}\text { Os profissionais são orientados à execução de pequenos procedimentos } \\
\text { demandados de maneira prática (tais como: liberação de medicamentos). }\end{array}$ & 1 & 2 & 3 & 4 & 5 \\
\hline Eficácia & $7 a$ & $\begin{array}{l}\text { Os profissionais estão lotados em departamentos conforme suas especiali- } \\
\text { zações profissionais, não havendo desvio de cargos técnicos na unidade. }\end{array}$ & 1 & 2 & 3 & 4 & 5 \\
\hline $\begin{array}{l}\text { Efetivi- } \\
\text { dade }\end{array}$ & $8 a$ & $\begin{array}{l}\text { A unidade se atenta para o desenvolvimento de indicadores qualitativos } \\
\text { junto aos usuários do serviço, a fim de identificar-se o nível de serviço e a } \\
\text { satisfação dos pacientes e acompanhantes. }\end{array}$ & 1 & 2 & 3 & 4 & 5 \\
\hline $\begin{array}{l}\text { Centrali- } \\
\text { dade no } \\
\text { Paciente }\end{array}$ & $\begin{array}{l}9 a \mathrm{e} \\
10 \mathrm{a}\end{array}$ & $\begin{array}{l}\text { Os profissionais são orientados quanto à assistência integral aos usuários, } \\
\text { no que se refere ao fornecimento de apoio técnico e moral durante as } \\
\text { diversas etapas da prestação de serviços. }\end{array}$ & 1 & 2 & 3 & 4 & 5 \\
\hline \multirow{2}{*}{$\begin{array}{l}\text { Segu- } \\
\text { rança }\end{array}$} & $11 a$ & $\begin{array}{l}\text { São ofertados treinamentos à equipe de assistência médica direcionados à atua- } \\
\text { lização profissional no que se refere ao cuidado com a segurança do paciente. }\end{array}$ & 1 & 2 & 3 & 4 & 5 \\
\hline & $12 a$ & $\begin{array}{l}\text { A equipe profissional (equipe de assistência médica e equipe de aten- } \\
\text { dimento) trabalha de maneira integrada, permitindo-se que o fluxo de } \\
\text { informações flua nos diversos estágios da prestação de serviços. }\end{array}$ & 1 & 2 & 3 & 4 & 5 \\
\hline \multirow{4}{*}{ Empatia } & $13 a$ & $\begin{array}{l}\text { A equipe de assistência médica é orientada ao desenvolvimento de aten- } \\
\text { dimento personalizado aos usuários, ainda que para isso seja necessário } \\
\text { maior tempo de atendimento. }\end{array}$ & 1 & 2 & 3 & 4 & 5 \\
\hline & $13 b$ & $\begin{array}{l}\text { A equipe de assistência médica é orientada acerca da demanda prevista } \\
\text { (quando é possível a previsão) de usuários a serem assistidos no expedien- } \\
\text { te de trabalho diário. }\end{array}$ & 1 & 2 & 3 & 4 & 5 \\
\hline & $14 a$ & A equipe administrativa é orientada ao tratamento cordial aos usuários. & 1 & 2 & 3 & 4 & 5 \\
\hline & $14 b$ & $\begin{array}{l}\text { A equipe administrativa possui conhecimentos técnicos necessários para } \\
\text { esclarecimento de dúvidas dos usuários. }\end{array}$ & 1 & 2 & 3 & 4 & 5 \\
\hline \multirow[b]{2}{*}{ Acesso } & $15 a$ & $\begin{array}{l}\text { Os profissionais são orientados à propiciação de menor tempo de espera } \\
\text { aos usuários. }\end{array}$ & 1 & 2 & 3 & 4 & 5 \\
\hline & $16 a$ & $\begin{array}{l}\text { Os profissionais buscam atuar com agilidade nos setores da unidade que } \\
\text { possuem a interface "urgência/emergência", de maneira que possibilite o } \\
\text { fluxo prioritário do atendimento. }\end{array}$ & 1 & 2 & 3 & 4 & 5 \\
\hline \multirow{7}{*}{$\begin{array}{l}\text { Legisla- } \\
\text { ção }\end{array}$} & $17 a$ & $\begin{array}{l}\text { Não há descriminação de qualquer natureza para efetivação de atendi- } \\
\text { mento e/ou agendamento para utilização dos serviços de saúde ofertados } \\
\text { pela unidade. }\end{array}$ & 1 & 2 & 3 & 4 & 5 \\
\hline & $18 \mathrm{a}$ & $\begin{array}{l}\text { Há igualdade na assistência à saúde. Assim, os usuários são atendidos sem } \\
\text { preconceitos ou privilégios de qualquer natureza, salvo as prioridades } \\
\text { estabelecidas em lei. }\end{array}$ & 1 & 2 & 3 & 4 & 5 \\
\hline & $19 a$ & São oferecidos serviços preventivos e curativos em caráter integral. & 1 & 2 & 3 & 4 & 5 \\
\hline & $20 a$ & A autonomia dos pacientes e acompanhantes é respeitada e estimulada. & 1 & 2 & 3 & 4 & 5 \\
\hline & $21 \mathrm{a}$ & $\begin{array}{l}\text { A unidade de atendimento se atenta para divulgação quanto aos tipos de } \\
\text { serviços de saúde ofertados na instituição. }\end{array}$ & 1 & 2 & 3 & 4 & 5 \\
\hline & $22 a$ & $\begin{array}{l}\text { A unidade dispõe de material legislativo que contém os princípios e as di- } \\
\text { retrizes do SUS (Sistema Único de Saúde), o qual se encontra à disposição } \\
\text { dos profissionais atuantes na instituição. }\end{array}$ & 1 & 2 & 3 & 4 & 5 \\
\hline & $22 b$ & $\begin{array}{l}\text { Os profissionais são orientados ao estudo das normas regulamentadoras } \\
\text { do SUS ao iniciarem suas atividades profissionais à serviço da unidade e/ } \\
\text { ou ao início de uma nova gestão. }\end{array}$ & 1 & 2 & 3 & 4 & 5 \\
\hline
\end{tabular}




\begin{tabular}{|c|c|c|c|c|c|c|c|}
\hline \multicolumn{8}{|c|}{ Importância de cada dimensão para a efetivação de serviço qualitativo no setor público de saúde } \\
\hline $\begin{array}{l}\text { Tangibi- } \\
\text { lidade }\end{array}$ & 1 & $\begin{array}{l}\text { Característica do que é tangível, palpável, que pode ser tocado (estrutura } \\
\text { do local, objetos etc.). }\end{array}$ & 1 & 2 & 3 & 4 & 5 \\
\hline $\begin{array}{l}\text { Eficiên- } \\
\text { cia }\end{array}$ & 2 & $\begin{array}{l}\text { Realizar os procedimentos com o mínimo de perda, por meio do melhor } \\
\text { uso possível do tempo, dos materiais e dos recursos humanos. }\end{array}$ & 1 & 2 & 3 & 4 & 5 \\
\hline Eficácia & 3 & Realizar os procedimentos da maneira correta. & 1 & 2 & 3 & 4 & 5 \\
\hline $\begin{array}{l}\text { Efetivi- } \\
\text { dade }\end{array}$ & 4 & Realizar os procedimentos de maneira certa e com qualidade & 1 & 2 & 3 & 4 & 5 \\
\hline $\begin{array}{l}\text { Centrali- } \\
\text { dade no } \\
\text { Paciente }\end{array}$ & 5 & $\begin{array}{l}\text { Parceria entre profissionais e pacientes a fim de garantir que o cuidado } \\
\text { respeite as necessidades e preferências dos pacientes. }\end{array}$ & 1 & 2 & 3 & 4 & 5 \\
\hline $\begin{array}{l}\text { Segu- } \\
\text { rança }\end{array}$ & 6 & $\begin{array}{l}\text { A prestação de serviço transmite a segurança de que o procedimento será } \\
\text { realizado sem danos aos pacientes. }\end{array}$ & 1 & 2 & 3 & 4 & 5 \\
\hline Empatia & 7 & $\begin{array}{c}\text { Ajudar e buscar compreender as necessidades do paciente, se colocando } \\
\text { no lugar dele e agindo segundo princípios morais. }\end{array}$ & 1 & 2 & 3 & 4 & 5 \\
\hline Acesso & 8 & $\begin{array}{c}\text { Tempo de espera e agilidade no atendimento em todos os setores da } \\
\text { unidade. }\end{array}$ & 1 & 2 & 3 & 4 & 5 \\
\hline $\begin{array}{l}\text { Legisla- } \\
\text { ção }\end{array}$ & 9 & $\begin{array}{c}\text { Conformidade com a lei, igualdade, imparcialidade, moderação, justiça, } \\
\text { equivalência. }\end{array}$ & 1 & 2 & 3 & 4 & 5 \\
\hline
\end{tabular}

Recebido: 11 jan. 2020

Aprovado: 17 nov. 2020

DOI: 10.20985/1980-5160.2020.v15n3.1605

Como citar: Amoury Neta, A.S.; Ferreira, B.C.S.; Bezerra, R.R.R.; Silva, L.B.P. (2020). Proposta de modelo de avaliação da qualidade direcionado ao Sistema Único de Saúde. Revista S\&G 15, 3, 250-262. https://revistasg. emnuvens.com.br/sg/article/view/1605 